\title{
AUTOPERCEPÇÃO DAS CONDIÇÕES DE SAÚDE BUCAL EM ADULTOS DO SUL DO BRASIL
}

\author{
Roberta Vedana Erckmann* \\ Mirian Kuhnen* \\ Everley Rosane Goetz ${ }^{\text {*t* }}$ \\ Anelise Viapiana Masiero ${ }^{*+x+\infty}$
}

RESUMO: Os agravos de saúde bucal ainda se constituem como importante problema de saúde pública. Estudos de base populacional são essenciais para identificar grupos prioritários e, neste contexto, investigar a autopercepção dos indivíduos complementa a análise objetiva, dando outro significado para as ações de planejamento em saúde. Este estudo teve como objetivo analisar a autopercepção de saúde bucal e fatores associados na população adulta de uma Unidade Básica de Saúde com Estratégia de Saúde da Família, do sul do Brasil. Participaram do estudo 207 sujeitos. A coleta de dados deu-se por meio de entrevista subjetiva, utilizando de questionário Oral Health Impact Profile - versão reduzida e exame clínico. Na análise dos resultados identificou-se que $43,0 \%$ da população avaliaram como positiva sua saúde bucal. Observou-se associação das variáveis e idade $(p=0,036)$, cor da pele $(p=0,030)$ e escolaridade $(p=0,022)$ com a autopercepção positiva. Em relação à necessidade de tratamento, 71,5\% acreditam precisar de alguma intervenção odontológica. Quando questionados sobre 0 impacto das condições bucais em sua qualidade de vida a maioria não percebe esta relação. No exame clínico, constatou-se que 48,2\% dos indivíduos possuíam cárie dental e $81,7 \%$ necessitavam de prótese. Nesse sentido é necessário dar ênfase à educação em saúde a fim de que a população perceba a relação da saúde bucal com a saúde geral e com a qualidade de vida.

PALAVRAS-CHAVE: Autopercepção; Saúde bucal; Qualidade de vida.

\section{SELF-PERCEPTION IN ORAL HEALTH CONDITIONS IN ADULTS, IN SOUTH BRAZIL}

\begin{abstract}
Oral health is still an important issue in public health. Population-based studies are relevant to identify priority groups and the investigation of people's self-perception within the context complement objective analysis, with other meanings for planned health activities. Self-perception of oral health and associated factors in adults of a Basic Health Unit coupled to Family Health Strategy in south Brazil are investigated. Further, 207 subjects participated and data were collected by a subjective interview (Oral Health Impact Profile questionnaire - a concise version and clinical exam). Results identified that $43.0 \%$ of the population under analysis evaluated their mouth health as positive. There was an association between age $(p=0.036)$, color $(p=0.030)$ and schooling $(p=0.022)$, with positive self-perception. When asked about the need for treatment, $71.5 \%$ answered that they required dental intervention. Most failed to perceive the relationship when questioned on the impact of mouth conditions on their life quality. Clinical examination revealed that $48.2 \%$ had teeth caries and $81.7 \%$ required prosthesis. Health education should be underscored so that the population perceives the relationship between mouth health and general health and life quality.
\end{abstract}

KEYWORDS: Self-perception; Oral health; Life quality.

\footnotetext{
Graduada em Odontologia, pela Universidade do Planalto Catarinense; Especialista em Residência Multiprofissional em Saúde da Família e Comunidade pela Universidade do Planalto Catarinense, Brasil.

** Mestre em Saúde Coletiva pelo Programa de Pós-graduação em Saúde Coletiva, da Universidade do Planalto Catarinense; Docente titular da disciplina de odontologia social e preventiva do curso de odontologia da Uniplac, Brasil.

${ }^{\star * \star}$ Doutora em Psicologia, pela Universidade Federal de Santa Catarina; Docente das Faculdades lesgo (Instituto Superior de Goiás) e IESB (Instituto Superior de Brasília), Brasil.

${ }^{\star * \star *}$ Doutora em Odontologia, pela Universidade de São Paulo. Docente do Programa de Pós-graduação em Ambiente e Saúde da Uniplac, Brasil. E-mail: avmasiero@gmail.com
} 


\section{INTRODUÇÃo}

0 último levantamento epidemiológico nacional identificou diminuição nos índices de dentes cariados e perdidos na população adulta, entretanto, observase que a morbidade da doença permanece inalterada (BRASIL, 2012). Apesar de os dados epidemiológicos serem fundamentais para planejamentos, organizações e monitoramento dos serviços de saúde são restritos à visão profissional objetiva e ganham outro significado quando acompanhados da avaliação da autopercepção das condições de saúde bucal, conferidas pelos próprios indivíduos, sobretudo considerando que 0 comportamento das pessoas é condicionado por suas percepções e pela importância atribuídas a ela (HAIKAL et al., 2011).

Nesse sentido, a fim de identificar grupos prioritários, formular e avaliar políticas públicas, 0 Ministério da Saúde em conjunto com a Fundação Instituto Brasileiro de Geografia e Estatística (IBGE) realizaram a Pesquisa Nacional de Saúde, em 2013, utilizando como um dos parâmetros, a autoavaliação de saúde (IBGE, 2017).

$A$ inserção da equipe de saúde bucal na Estratégia Saúde da Família (ESF), a partir de 2000 (BRASIL, 2000), ampliou o acesso aos serviços de saúde. A despeito dos princípios do SUS de universalidade, integralidade e equidade, os indicadores de saúde bucal não têm mostrado resultados tão bons do modelo de atenção quanto os indicadores de mortalidade infantil, desnutrição de internações por causas preveníveis (PEREIRA et al., 2012).

Considera-se, portanto, que 0 estudo da autopercepção em população assistida pela ESF constitui uma ferramenta importante para 0 planejamento das ações de saúde bucal, no intuito de tornar os serviços mais resolutivos. Neste contexto, 0 presente estudo teve como objetivo, analisar a autopercepção de saúde bucal em adultos de um município do sul do Brasil.

\section{MATERIAL E MÉTODOS}

0 estudo caracteriza-se como transversal exploratório com abordagem quantitativa. A pesquisa foi aprovada pelo Comitê de Ética em Pesquisa da Universidade do Planalto Catarinense, segundo protocolo 047-13.

A amostra foi obtida por conveniência durante 0 período de cinco meses. Foram convidados a participar pacientes cadastrados na área de abrangência de uma Unidade de Saúde da Família (USF) onde estavam inseridos residentes da Residência Multiprofissional em Saúde da Família e Comunidade, de um município de médio porte do sul do Brasil. 0 município possui 27 USF, 41 equipes de Estratégia da Saúde da Família e 35 equipes de saúde bucal (LAGES, 2017).

Considerou-se como critérios de inclusão: pacientes com idade entre 18 anos e 60 anos, de ambos os sexos, funcionalmente independentes e que aceitaram participar da pesquisa.

Os dados foram coletados durante a consulta odontológica na USF ou nas visitas domiciliares. Os procedimentos de coleta de dados incluíram: entrevista estruturada referente aos dados socioeconômicos e ao uso de serviços odontológicos (BEDIN, 2012), a percepção de necessidade de tratamento bem como autopercepção de sua saúde bucal (SOARES et al., 2011). Por fim, realizou-se 0 exame clínico para avaliar as reais condições de saúde e a necessidade de tratamento. 0 exame clínico foi feito por único examinador devidamente treinado, utilizando luz artificial, sonda exploradora número 5 , espelho bucal plano, sonda periodontal.

As variáveis incluídas na avaliação e questionário tinham como objetivo detectar a autopercepção da pessoa sobre sua própria saúde bucal, enquanto no exame clínico as variáveis tinham como objetivo avaliar clinicamente a condição bucal e as condições dos tecidos moles, presença ou ausência de cárie, presença ou ausência de dentes, presença ou ausência de doença periodontal, necessidade de prótese.

A avaliação da autopercepção de saúde bucal 
foi obtida por meio da pergunta: Como você considera sua saúde bucal? Sendo as opções de resposta os itens "Excelente", "Muito boa", "Boa", "Regular" e "Ruim". Durante a análise as categorias: "Excelente", "Muito boa" e "Boa" integraram a autopercepção positiva e as categorias: "Regular" e "Ruim", foram consideradas como autopercepção de saúde bucal negativa. Em relação aos dados socioeconômicos, a idade foi coletada, em anos completos e, posteriormente, categorizada de acordo com as seguintes faixas etárias 18-29, 30-39, 40-49 e 50-59 anos (BEDIN, 2012). A escolaridade foi coletada em série estudada e depois categorizada em não alfabetizado, ensino fundamental, médio e superior (BEDIN, 2012). A renda familiar foi categorizada em até um salário; de 1,1 a 3 salários; de 3,1 a 5 salários; e, 5,1 ou mais (BEDIN, 2012). A cor da pele autorreferida foi investigada, segundo as categorias adotadas pelo Instituto Brasileiro de Geografia e Estatística (IBGE, 2017): branca, preta, parda, amarela (asiáticos) e indígena. Os hábitos e comportamentos relacionados à saúde foram classificados em uso de tabaco, álcool e outras drogas (SILVA; FERNANDES, 2001).

Foi utilizada uma versão simplificada do Oral Health Impact Profile, 0 OHIP-14, conforme preconizado por SLADE (1997) o qual possui sete categorias de análises, sendo as dimensões, limitação funcional, dor física, desconforto psicológico, desabilidade física, desabilidade psicológica, desabilidade social e limitação física.

As respostas do OHIP-14 foram classificadas de forma dicotômica, sendo que aos itens: "sempre" e "quase sempre" foi atribuído valor 1 , considerando existência de impacto na qualidade de vida. As respostas "às vezes", "nunca" e "quase nunca" foram consideradas como inexistência do impacto da condição de saúde bucal na qualidade de vida do indivíduo, recebendo 0 valor 0 . Cada resposta teve um peso, o qual foi somado ao final do questionário. A soma dos pontos representou o impacto da saúde bucal na qualidade de vida dos adultos, e quanto mais alta a soma maior 0 impacto negativo (SLADE, 1997).

Os dados foram analisados pelo programa estatístico Statistical Package for the Social Sciences
(SPSS), versão 21. Inicialmente foi realizada a estatística descritiva das variáveis. Para verificar as associações foi usado o teste de qui-quadrado com nível de significância de 0,05.

\section{RESULTADOS}

Participaram do estudo 207 indivíduos. Destes, $167(80,7 \%)$ eram mulheres e 40 eram homens (19,3\%). Quanto à autopercepção de saúde bucal, os dados demostraram que $43,0 \%$ dos entrevistados avaliaram como positiva sua saúde bucal (Tabela 1).

Tabela 1. Distribuição da população, segundo critérios de autopercepção de saúde bucal

\begin{tabular}{lcc}
\hline Autopercepção & $\mathbf{n}$ & \% \\
\hline Ruim & 32 & 15,5 \\
Regular & 86 & 41,5 \\
Boa & 81 & 39,1 \\
Muito boa & 6 & 2,9 \\
Excelente & 2 & 1,0 \\
\hline Total & $\mathbf{2 0 7}$ & $\mathbf{1 0 0}$ \\
\hline
\end{tabular}

Fonte: elaborada pelos autores.

Observou-se associação das variáveis autopercepção das condições de saúde bucal e idade $(p=0,036)$, cor da pele $(p=0,030)$ e escolaridade $(p=0,022)$ com a autopercepção positiva. Pessoas que se autorreferenciam de cor branca, maior nível de escolaridade e mais velhos, tendem a perceber positivamente sua saúde bucal (Tabela 2). Em relação à autopercepção de necessidade de tratamento odontológico 71,5\% afirmaram necessitar de tratamento (Tabela 3). 
Tabela 2. Autopercepção positiva das condições de saúde bucal, segundo características demográficas e socioeconômicas em adultos de um município de médio porte da serra catarinense

\begin{tabular}{|c|c|c|}
\hline \multicolumn{2}{|c|}{ Autopercepção positiva da saúde } & \multirow{2}{*}{$\begin{array}{c}\text { Todos }(\mathrm{n}=207) \\
\%\end{array}$} \\
\hline & $\mathbf{n}$ & \\
\hline Variáveis & $(n=89)$ & \\
\hline \multicolumn{3}{|l|}{ Gênero } \\
\hline Homens $(n=40)$ & 18 & $45 \%$ \\
\hline Mulheres $(n=167)$ & 71 & $42 \%$ \\
\hline$p$ & 0,389 & \\
\hline \multicolumn{3}{|l|}{ Cor da pele } \\
\hline Brancos $(n=120)$ & 62 & $51,6 \%$ \\
\hline Indígenas $(\mathrm{n}=3)$ & 1 & $33,3 \%$ \\
\hline Negro $(n=16)$ & 6 & $37,5 \%$ \\
\hline Pardo $(\mathrm{n}=68)$ & 20 & $29,4 \%$ \\
\hline$p$ & $0,030^{*}$ & \\
\hline \multicolumn{3}{|l|}{ Renda familiar } \\
\hline Até 1 salário $(\mathrm{n}=95)$ & 56 & $58,9 \%$ \\
\hline 1,1 a 3 salários $(n=84)$ & 31 & $36,9 \%$ \\
\hline 3,1 a 5 salários $(n=25)$ & 1 & $0,4 \%$ \\
\hline 5,1 ou mais $(3)$ & 1 & 33,3 \\
\hline$p$ & 0,74 & \\
\hline \multicolumn{3}{|l|}{ Escolaridade } \\
\hline Não alfabetizado ( $n=11)$ & 4 & $36,6 \%$ \\
\hline Fundamental $(n=121)$ & 54 & $44,6 \%$ \\
\hline Médio ( $\mathrm{n}=58)$ & 20 & $34,4 \%$ \\
\hline Superior $(n=17)$ & 11 & $64,0 \%$ \\
\hline$p$ & $0,022^{*}$ & \\
\hline \multicolumn{3}{|l|}{ Hábitos } \\
\hline Tabaco $(n=42)$ & 17 & $40,4 \%$ \\
\hline Álcool $(n=16)$ & 7 & $43,7 \%$ \\
\hline Tabaco e álcool (n=12) & 5 & $41,6 \%$ \\
\hline Outros $(\mathrm{n}=3)$ & 1 & $33,3 \%$ \\
\hline Nenhum (134) & 59 & $44,0 \%$ \\
\hline$p$ & 0,432 & \\
\hline \multicolumn{3}{|l|}{ Idade } \\
\hline 18 a 29 anos $(n=66)$ & 22 & $33,3 \%$ \\
\hline 30 a 39 anos $(n=46)$ & 18 & $39,1 \%$ \\
\hline 40 a 49 anos $(n=41)$ & 18 & $43,9 \%$ \\
\hline 50 a 59 anos $(n=54)$ & 31 & $57,4 \%$ \\
\hline$p$ & $0,036^{*}$ & \\
\hline
\end{tabular}

Tabela 3. Distribuição da população, segundo autopercepção de necessidade tratamento odontológico

\begin{tabular}{lcc}
\hline Autopercepção & N & \% \\
\hline Não & 59 & 28,5 \\
Sim & 148 & 71,5 \\
\hline Total & $\mathbf{2 0 7}$ & $\mathbf{1 0 0}$ \\
\hline
\end{tabular}

Fonte: elaborada pelos autores.

Por meio do exame clínico, constatou-se que $48,2 \%$ dos indivíduos possuíam cárie dental, 50,7\% cálculo dental, 43,0\% gengivite, $14,5 \%$ raiz residual e $5,3 \%$ agravos de tecidos moles.

Analisando 0 uso e a necessidade de prótese dental, verificou-se que $27,1 \%$ dos pacientes usavam prótese total e 16,4\% usavam prótese parcial removível, 16,9\% necessitavam de prótese total e 64,8\% necessitavam de prótese parcial removível.

Em relação ao uso de serviços odontológicos, $58 \%$ dos entrevistados relataram utilizar somente os serviços do SUS e $53,6 \%$ realizaram consulta odontológica a menos de um ano.

Quando questionados sobre o motivo da última visita ao dentista, $55,1 \%$ foram à consulta de rotina e $35,3 \%$ porque estavam sentindo dor. Em relação ao tratamento realizado nesta consulta, $32,9 \%$ dos entrevistados realizaram restauração e 19,3\% extração.

Verificou-se que $80,7 \%$ dos entrevistados já realizaram exodontia de pelo menos um dente ao longo da vida, destes $61,4 \%$ por cárie.

Tabela 4. Distribuição segundo OHIP 14

\begin{tabular}{ccc}
\hline Soma das respostas $\mathbf{~ O H I P - 1 4}$ & $\mathbf{N}$ & $\mathbf{\%}$ \\
\hline 0 & 119 & 57,5 \\
1 & 21 & 10,1 \\
2 & 19 & 9,2 \\
3 & 13 & 6,3 \\
4 & 10 & 4,8 \\
5 & 8 & 3,9 \\
6 & 2 & 1,0 \\
\hline 7 & 4 & 1,9 \\
\hline 8 & 3 & 1,4 \\
9 & 3 & 1,4 \\
10 & 2 & 1 \\
\hline 11 & 2 & 1 \\
\hline 12 & 1 & 5 \\
\hline Total & $\mathbf{2 0 7}$ & $\mathbf{1 0 0}$ \\
\hline
\end{tabular}

Nota: * Valor mínim0 $=0$; valor máximo $=14$

Fonte: elaborada pelos autores

\section{DISCUSSÃO}

É evidente a influência de fatores micro e macrodimensionais com repercussões na autopercepção de saúde bucal e na qualidade 
de vida das pessoas. Assim, são importantes as investigações que associem a autopercepção de saúde bucal das pessoas com a autopercepção de necessidade de tratamento odontológico, as condições socioeconômicas, sociodemográficas, tabagismo, consumo de álcool ou outras drogas e impacto da qualidade de vida (GABARD0 et al., 2013).

Em relação à autopercepção das condições de saúde, $43,0 \%$ da população estudada autoperceberam sua saúde bucal positiva, dado compatível com a Pesquisa Nacional de Saúde Bucal, 2010, que identificou a autopercepção positiva em $40,3 \%$ dos adultos participantes (BRASIL, 2012). No inquérito realizado neste mesmo município, em 2007, a prevalência de autopercepção positiva foi de 56,8\% (KUHNEN et al., 2009). Índices superiores foram encontrados por Santos et al. (2016), ficando em torno de $75 \%$ de autopercepção positiva.

$\mathrm{Na}$ análise das variáveis socioeconômicas e demográficas houve diferenças significantes para idade, escolaridade e cor da pele. Indivíduos com maior faixa etária, maior nível de escolaridade e que autorreferem cor da pele branca, apresentaram tendência de avaliar positivamente sua saúde bucal. A análise dos dados da Pesquisa Nacional de Saúde identificou correlação das condições socioeconômicas com a autopercepção de saúde, reportando que indivíduos mais jovens, de cor branca, com maior grau de instrução e moradores da zona urbana, autopercebem de forma mais positiva sua condição de saúde bucal (NICO et al., 2015).

No que se refere à renda, não se identificou significância estatística. Embora a literatura tem mostrado que 0 avanço da idade influencia a percepção bucal negativa (GABARDO et al., 2013), no presente estudo os mais velhos percebem de forma mais positiva suas condições de saúde bucal. Em recente estudo de revisão de literatura, observou-se que os fatores sociodemográficos como o gênero e 0 estado civil influenciam a percepção da saúde bucal mais do que a escolaridade e a idade (ROSENDO et al., 2017).

As variáveis relacionadas a hábitos deletérios como tabagismo, consumo de álcool ou outras drogas não apresentaram relação com a autopercepção de saúde bucal. Segundo Kuhnen et al. (2009), pessoas que autoavaliaram sua saúde bucal como negativa e que possuem problemas com álcool apresentam maior prevalência de tabagismo.

Vale salientar que $71,5 \%$ dos adultos entrevistados autoperceberam a necessidade de tratamento odontológico. No exame clínico observouse que em torno de $50 \%$ da população, de fato, necessitavam de algum tipo de tratamento para cárie, doença periodontal e outros agravos. Apesar desta concordância, entre autopercepção e diagnóstico clínico, em muitos casos, os critérios utilizados pelos indivíduos para determinar sua necessidade de tratamento são diferentes daqueles utilizados pelo profissional. Enquanto o cirurgião-dentista avalia a condição de saúde a partir da ausência ou presença de doença, o paciente parece priorizar mais a estética e necessidades funcionais do que as condições de saúde relacionadas com a doença (SANTOS et al., 2016).

Contudo, a grande maioria dos indivíduos relatou não sofrer impacto na qualidade de vida com problemas bucais. Isto denota que os indivíduos não conseguem depreender a relação entre a saúde bucal e saúde geral, como se estas não estivessem conectadas.

Avaliando a percepção do usuário sobre a necessidade de prótese, a maioria dos entrevistados não autopercebeu a necessidade da mesma. Esta percepção subestimada em relação à necessidade de próteses, totais ou parciais, foi identificada por Nascimento et al. (2016) na análise de fatores associados à concordância entre autopercepção e avaliação clínica de necessidade de tratamento utilizando como fonte de dados o SBBrasil 2010 e 0 SBMinas Gerais, 2012.

Na atualidade, o termo "qualidade de vida" relacionado diretamente à saúde bucal tem sido utilizado para reconhecer o impacto das doenças bucais na vida cotidiana das pessoas (VASCONCELOS et al., 2012). E dentre os instrumentos de avaliação do impacto das condições de saúde na qualidade de vida, 0 questionário OHIP-14 tem sido 0 mais amplamente utilizado (GABARD0 et al., 2013) 
Com relação à análise do impacto das condições bucais na qualidade de vida, $57,5 \%$ da população estudada obtiveram somatório 0 na análise do OHIP14, 0 que significa não perceber impacto algum. Ainda, a média geral do somatório total do OHIP-14 foi baixa $(1,12)$, corroborando com Guerra et al., (2014) que sugerem que estes resultados associem-se ao acesso e uso dos serviços odontológicos.

Assim, parece evidente que a percepção da condição bucal e a importância dada a ela condicionam o comportamento do indivíduo. Mais de $30 \%$ dos entrevistados procuraram o serviço apenas quando tiveram dor 0 que sinaliza a preferência da utilização dos serviços odontológicos para tratamentos curativos em detrimento de procedimentos preventivos (SANTOS et al., 2016).

Na maioria das vezes a razão para as pessoas não procurarem 0 atendimento odontológico é a não percepção de suas necessidades. Pressupõe-se que a identificação da percepção da condição bucal do indivíduo pode ser o primeiro passo para a elaboração de programas que incluam ações educativas e de promoção voltadas para o autocuidado, além de ações preventivas e reabilitadoras (SILVA; FERNANDES, 2001).

Ademais, as avaliações subjetivas devem ser interpretadas como aportes críticos essenciais aos indicadores clínicos, auxiliando na identificação de grupos vulneráveis que precisam de intervenções complexas e, muitas vezes, individualizadas (GABARD0 et al., 2013)

É pertinente destacar o papel da Residência Multiprofissional em Saúde da Família e Comunidade, visto que ela possibilita a formação de profissionais com olhar ampliado para o contexto social e familiar da população assistida, objetivando a integralidade do cuidado. Como limitação do estudo, destaca-se que os dados ora apresentados demonstram características específicas de um grupo estudado e não podem ser extrapolados para o restante da população. Entretanto, contribuem para o planejamento das ações de saúde bucal da população assistida pela ESF, destacando a importância de se considerar autopercepção de saúde bucal e seu impacto na qualidade de vida, de forma que os serviços sejam mais resolutivos, levando em conta a necessidade de cada indivíduo.

Nesse sentido, a educação em saúde tem papel fundamental na promoção, prevenção e reabilitação da cavidade bucal a fim de proporcionar melhor autonomia e qualidade de vida (ROSEND0 et al., 2017). Entretanto, para que na prática estes objetivos se concretizem, a Residência Multiprofissional em Saúde da Família e Comunidade caracteriza-se como espaço propício para o fortalecimento da articulação ensino/ serviço, para inserção do trabalho interprofissional nos cenários pedagógicos e organizacionais, como estratégia nos processos de formação e de trabalho em saúde.

Assim, para estudos futuros, sugere-se a análise da autopercepção das condições de saúde bucal de indivíduos assistidos por áreas com cobertura da RMSFC em comparação a indivíduos pertencentes a áreas não assistidas pela RMSFC.

\section{CONCLUSÕES}

Considerando as condições do presente estudo é possível concluir que a maioria dos participantes percebe de forma negativa sua condição de saúde bucal apesar de não relatarem impacto na qualidade de vida, sendo que os participantes que se autorreferenciam de cor branca, mais escolarizados e mais velhos, perceberam de forma mais positiva sua saúde bucal. Por fim, dos entrevistados, mais da metade relatou que as suas condições de saúde bucal não impactam na qualidade de vida.

\section{AGRADECIMENTOS}

Agradecimentos aos residentes, Bruna Damasceno da Silva e Claudio Juliano Cruz Fontanella, que contribuíram com a coleta de dados. 


\section{REFERÊNCIAS}

BEDIN, E. Autopercepção e condições de saúde bucal em adultos e idosos Itapuca/RS. 2012. Tese (Doutorado) - Universidade Santa Cecília, 2012.

BRASIL. Ministério da Saúde. Portaria $n^{0}$. 1.444/GM. Cria 0 incentivo de Saúde Bucal para o financiamento de ações e da inserção de profissionais de Saúde Bucal no Programa de Saúde da Família. Diário Oficial da União, 2000.

BRASIL. Ministério da Saúde. SB Brasil 2010: Pesquisa Nacional de Saúde Bucal: resultados principais. Secretaria de Atenção à Saúde. Secretaria de Vigilância em Saúde. Brasília: 2012.116 p.

GABARDO, M. C. L.; MOYSÉS, S. T.; MOYSÉS, S. J. Autopercepção de saúde bucal conforme 0 Perfil de Impacto da Saúde Bucal (OHIP) e fatores associados: revisão sistemática. Revista Panamericana de Salud Publica, v. 33, n. 6, p. 439-445, 2013.

GUERRA, M. J. et al. Impacto das condições de saúde bucal na qualidade de vida de trabalhadores. Ciência \& Saúde Coletiva, v. 19, n. 12, p. 4777-4786, 2014.

HAIKAL, D. et al. Autopercepção da saúde bucal e impacto na qualidade de vida do idoso: uma abordagem quanti-qualitativa. Ciência \& Saúde Coletiva, v. 16, n. 7, p. 3317-3328, 2011.

IBGE. Instituto Brasileiro de Geografia e Estatística. Estimativas de população. 2017. Disponível em: <http://www.ibge.gov.br/home/estatistica/populacao/ estimativa2017/default.shtm>. Acesso em: 2017.

KUHNEN, M. et al. Tabagismo e fatores associados em adultos: um estudo de base populacional. Revista Brasileira de Epidemiologia, v. 12, n. 4, p. 615-626, 2009.

LAGES. Secretaria de Saúde. Dados atenção básica. Departamento de atenção básica. 2017.

NASCIMENTO, A. R. D.; ANDRADE, F. B. D.; CÉSAR, C. C. Fatores associados à concordância entre autopercepção e avaliação clínica da necessidade de tratamento dentário em adultos do Brasil e de Minas Gerais. Cadernos de Saúde Pública, v. 32, n. 10, p. 1-13, 2016.

NICO, L. S. et al. Saúde Bucal autorreferida da população adulta brasileira: resultados da Pesquisa Nacional de Saúde 2013. Revista Ciência \& Saúde Coletiva, v. 21, n. 2, p. 389-398, 2016.

PEREIRA, C. R. D. S. et al. Impacto da Estratégia Saúde da Família sobre indicadores de saúde bucal: análise em municípios do Nordeste brasileiro com mais de 100 mil habitantes. Cadernos de Saúde Pública, v. 28, n. 3, p. 449-462, 2012.

ROSENDO, R. A. et al. Autopercepção de saúde bucal e seu impacto na qualidade de vida em idosos: uma revisão de literatura. Revista Saúde \& Ciência Online, v. 6, n. 1, p. 89-102, 2017.

SANTOS, L. M. et al. Autopercepção sobre saúde bucal e sua relação com utilização de serviços e prevalência de dor de dente. Revista Ciência Plural, v. 2, n. 2, p. 14-27, 2016.

SILVA, S. R. C; FERNANDES, R.A Autopercepção das condições de saúde bucal por idosos. Revista de Saúde Pública, v. 35, n. 4, p. 349-355, 2001.

SLADE, G. D. Derivation and validation of a shortform oral health impact profile. Community dentistry and oral epidemiology, v. 25, n. 4, p. 284-290, 1997.

SOARES, B.G. et al. Associação da autopercepção de saúde bucal com parâmetros clínicos orais. Revista brasileira de odontologia, v. 68 , ก. 2, p. 268-273, 2011.

VASCONCELOS, L. C. A. et al. Self-perceived oral health among elderly individuals in a medium-sized city in Northeast Brazil. Cadernos de Saúde Pública, v. 28, n. 6, p. 1101-1110, 2012.

Recebido em: 07 de Agosto de 2017 Aceito em: 19 de outubro de 2017 